\section{YEAST TECHNOLOGY}

\section{Yeast Technology}

By John White. Pp. xvi $+432+17$ plates. (London : Chapman and Hall, Ltd., 1954.) 55s. net.

$\mathrm{T}$ HIS volume makes a welcome addition to the already large literature of commercial yeast technology. The author states in his preface that it is largely the outcome of a series of papers communicated by him to various journals, and in its general make-up it certainly bears unmistakable traces of its origin. There is a good deal of needless repetition which could have been obviated by more careful editing.

The greater part of this book is concerned with the manufacture of bekers' yeast on a commercial scale, as well as the important questions of quality of the product and biological control. From a reading of these chapters it is obvious that the author is a master of his subject. They could not well be bettered, and should be studied by every beginner entering the field of commercial yeast production. Unfortunately, when the author strays from his chosen field his touch is by no means so sure. The chapters on yeast genetics and food and fodder yeasts, for example, should either be entirely omitted or very greatly expanded. The chapter on yeast genetics as it stands at present is too scrappy and inadequate to be of any real service to a beginner. The treatment of brewers' yeast is also not very adequate. The chapter on this subject has been contributed by Dr. C. Rainbow, and it is clear that he has been hampered by lack of space ; but he has certainly used the space he has been allowed to good purpose. In another edition this chapter should also be very greatly expanded. It would, for example, have been of great help to the practical brewer to be given more details of how to select his pitching yeasts and how to maintain his stock cultures in an active state.

The main purpose of this volume is to describe the commercial manufacture of bakers' yeast; and this is done with a wealth of detail in the early chapters and should be of the greatest value to beginner and experienced worker. The remaining chapters, apart from those on yeast genetics, food and fodder yeasts and brewers' yeasts, are concerned with such important subjects as yeast nutrients, structure and consistency of yeasts, aspects of yeast physiology and disposal of effluents from yeast factories. There are also chapters on the effect of aeration and temperature on yeast growth, and these will repay careful study.

The chapter on the microbiological control of yeast growth processes is particularly well done; but in another edition the author should give full details of how stock cultures of bakers' yeast are maintained and how fresh cultures are selected.

The information on the behaviour of bakers' yeast during panary fermentation is up to date and should be studied by every baker, even if he has only a mudimentary knowledge of chemistry. It may prevent him from laying the blame at the yeast manufacturer's door for his own mistakes.

The publishers are to be congratulated on the general make-up of this book; the quality and clearness of the various illustrations and diagrams are excellent and leave nothing to be desired. Apart from the criticisms which have been made-and they are all of a minor character-this book can be strongly recommended and should be acquired by everyone interested in this subject.

E. BARTON-WRIGHT

\section{COSMIC EVOLUTION}

Stars in the Making

By Cecilia Payne-Gaposchkin. Pp. xii $+160+67$ plates. (London : Eyre and Spottiswoode (Publishers), Ltd., 1953.) 25s. net.

WE early writings of Dr. Payne-Gaposchkin (then Miss Cecilia Payne) provided much of the empirical knowledge that was used to test the theoretical predictions made by the late E. A. Milne and his contemporaries in their classical researches on stellar atmospheres. She has since become a leading worker on variable stars. In the present book she ranges over a wider field and addresses herself to a wider class of readers than in her previous writings. In doing so she reveals a talent for brilliant and stimulating exposition.

Dr. Payne-Gaposchkin calls her theme the drama of cosmic evolution. In her first two chapters on "The Actors" she gives a vivid description of the physical variety of the stars and of the physical conditions of interstellar material. The next three chapters describe "The Scene" that is witnessed by astronomical observation. In the reviewer's opinion, this is the best part of the book and includes much information that has not before been presented in a popular account. It deals with the physical characteristics of double stars, clusters of stars and the strikingly different stellar sequences found in golactic and in globular clusters, with galaxies and, particularly informatively, with the comparative study of the Milky Way galaxy and the Andromeda nebula, and with Baade's two stellar populations and the varying manner of their occurrence in different galaxies. Most of the collection of sixty-odd splendid plates at the end of the book illustrate this section. Several important observational advances in the past two years would have permitted some statements to have been made in a more categorical form had they been available when the book was being written.

The "Drama" itself has to be inferred from the analysis of this scene. Dr. Payne-Gaposchkin marshals the inferences that can be made about the ages of various astronomical systems and then seeks to show that other general indications are compatible with these in suggesting a certain evolutionary sequence. Many of her ideas are in keeping with some current trends in astronomical thought (though she does retain the now generally rejected fission theory of the origin of double stars). However, one would wish to see the ideas brought into closer relationship with theories of stellar structure at one extreme, and with some theory of the formation of galaxies at the other, before venturing an opinion upon them.

Dr. Payne-Gaposchkin likens her undertaking to trying to reconstruct the story of a film from the array of still pictures displayed outside a cinema. Her own reconstruction will provoke lively curiosity - even if the display does not yet carry the announcement, "coming shortly".

W. H. MoCrea 